\title{
How to stop breathing: On the matter of getting respiratory motion under control
}

\author{
Dominik C. Benz, MD, a and Ronny R. Buechel, $\mathrm{MD}^{\mathrm{a}}$ \\ ${ }^{a}$ Cardiac Imaging, Department of Nuclear Medicine, University Hospital Zurich, Zurich, \\ Switzerland
}

Received May 6, 2016; accepted May 6, 2016

doi: $10.1007 / \mathrm{s} 12350-016-0542-\mathrm{z}$

\section{See related article, pp. 1598-1607}

Respiratory motion is a recognized potential source of artifacts in single-photon emission computed tomography myocardial perfusion imaging (SPECT MPI). Respiration leads to a complex three-dimensional movement of the heart predominantly in the craniocaudal axis, potentially introducing anterior and particularly inferior wall blurring in SPECT MPI. ${ }^{1}$ Among other techniques, respiratory gating has repeatedly been proposed to address the issue. ${ }^{2,3}$ However, with conventional SPECT gamma cameras, implementation of respiratory gating is difficult and cumbersome due to the inherent step-and-shoot acquisition of the rotating detectors. The introduction of gamma cameras with cadmium-zinc-telluride (CZT)-based semiconductor detector technology may facilitate detection and correction of respiratory motion due to the combination of a stationary camera design without the need of rotating the detectors around the patient, and list-mode data acquisition. The beneficial properties of CZT cameras have also paved the way for other approaches such as prospective respiratory triggering, where acquisition is only performed at a certain inspiration level (e.g., during inspiration breath-hold only) thus eliminating respiratory motion altogether. ${ }^{4,5}$ However, such an approach inevitably leads to complication and prolongation of acquisition protocols. This may prove problematic particularly in view of the current strive to reduce radiation dose exposure by lowering the isotope doses because the

Reprint requests: Ronny R. Buechel, MD, Cardiac Imaging, Department of Nuclear Medicine, University Hospital Zurich, Zurich, Switzerland; ronny.buechel@usz.ch

J Nucl Cardiol 2017;24:1608-9.

1071-3581/ $\$ 34.00$

Copyright (c) 2016 American Society of Nuclear Cardiology. latter inevitably leads to an increase in acquisition time. ${ }^{6}$ Thus, implementation of motion detection and correction algorithms may offer a more promising strategy for routinely controlling respiratory motion in nuclear cardiac imaging. These may come as software-based and purely data-driven solutions without any additional burden neither for the patient nor for the technician such as, for example, more complicated acquisition techniques, prolonged acquisition time hampering patient comfort and throughput, or even the need for increased radiotracer activity.

In the current issue of the Journal of Nuclear Cardiology ${ }^{\circledR}$, Daou et al demonstrate the feasibility of a recently introduced method for data-driven respiratorymotion correction for images acquired on a CZT SPECT camera. Their software by the name of Respiratory Gating (REGAT) retrospectively divides an entire SPECT dataset acquired in list-mode into short sequences of $500 \mathrm{~ms}$ duration each. Subsequently, these small datasets are then processed by a statistical method specifically adapted to handle low signal-to-noise ratio data, and a respiratory-motion curve is generated. Thereafter, the curve is filtered and-based on the amplitude of the curve-the software provides a mean respiratory-motion SPECT study which is then used for creating reconstructions either with or without realignment using REGAT. In their retrospective study, 18 patients who underwent stress/rest ${ }^{99} \mathrm{~m}$ Tc-Tetrofosmin SPECT MPI were included. Stress images were acquired 15 minutes after the injection of $2 \mathrm{MBq} \cdot \mathrm{kg}^{-1}{ }^{99 \mathrm{~m}} \mathrm{Tc}-$ Tetrofosmin. Three hours later, $6 \mathrm{MBq} \cdot \mathrm{kg}^{-1}$ of ${ }^{99 \mathrm{~m}} \mathrm{Tc}-$ Tetrofosmin was injected and followed by rest images after 45 minutes. Data were acquired in list-mode over 5 minutes for both stress and rest. No scatter or attenuation correction was performed. To evaluate the impact of the REGAT software, objective image characteristics were evaluated such as minimum left ventricular (LV) cavity counts, maximum LV myocardial counts, LV contrast as well as full width at half maximum of the 
anterior and inferior LV myocardial walls. In their study, mean motion detected was $9.7 \mathrm{~mm}$ in the cranio-caudal, $1.4 \mathrm{~mm}$ in the left-right arm, and $2.2 \mathrm{~mm}$ in the ventraldorsal axis. Of note, motion of more than $10 \mathrm{~mm}$ in the cranio-caudal axis was identified in a majority of patients. Regarding image quality, there was a positive impact of REGAT on all the measured image characteristics. Moreover, improvement correlated significantly with the amplitude of cranio-caudal motion indicating that with more extensive motion, the positive impact of respiratory-motion correction increases.

The authors deserve plenty of credit for their noteworthy pilot study demonstrating the feasibility of a novel method to correct the respiratory motion. Of note, the REGAT software not only seems to provide a substantial improvement in image quality, but also achieves this at minimal additional efforts by simply analyzing routinely acquired data. However, as the authors themselves state, some limitations exist and should be mentioned briefly. Firstly, as stress was induced by either exercise or dobutamine, breathing levels may not have completely normalized in all patients at the time of rest image acquisition starting 15 minutes later. This may have introduced an "upward creep" during image acquisition, ${ }^{7}$ potentially leading to an overestimation of real respiratory motion in the cranio-caudal axis. Thus, future studies will have to show if similar respiratory motion is observed after pharmacologically induced vasodilation. Secondly, the mean cumulated counts within the left ventricular myocardium for stress images were 0.9 million which is slightly below the commonly suggested 1 million counts threshold. ${ }^{8}$ This may have had a potential negative impact on image quality. The authors state that if cumulated counts obtained at stress were lower than the presumed critical threshold, we should not expect a high correlation between stress and rest respiratory-motion magnitudes. Although the authors' argument is arguably valid, the fact nevertheless may potentially limit the generalizability of their results. Finally, the REGAT software has not yet been validated for CZT SPECT cameras through comparison with a functional standard of reference. Although respiratory-motion curves extracted by REGAT from data acquired with a conventional SPECT camera have previously been shown to correlate well to respiratorymotion curves obtained by belts or video, future studies are needed to assure that this holds true for newer generation CZT cameras as well and, more importantly, if respiratory-motion correction indeed confers a further increase in diagnostic accuracy-thereby revealing its potential clinical benefit.

\section{References}

1. Pitman AG, Kalff V, Van Every B, Risa B, Barnden LR, Kelly MJ. Contributions of subdiaphragmatic activity, attenuation, and diaphragmatic motion to inferior wall artifact in attenuationcorrected Tc-99m myocardial perfusion SPECT. J Nucl Cardiol 2005;12:401-9.

2. Kovalski G, Keidar Z, Frenkel A, Sachs J, Attia S, Azhari H. Dual, "motion-frozen heart" combining respiration and contraction compensation in clinical myocardial perfusion SPECT imaging. J Nucl Cardiol 2009;16:396-404.

3. Cho K, Kumiata S, Okada S, Kumazaki T. Development of respiratory gated myocardial SPECT system. J Nucl Cardiol 1999;6:20-8.

4. Buechel RR, Pazhenkottil AP, Herzog BA, Husmann L, Nkoulou RN, Burger IA, et al. Real-time breath-hold triggering of myocardial perfusion imaging with a novel cadmium-zinc-telluride detector gamma camera. Eur $\mathrm{J}$ Nucl Med Mol Imaging 2010;37:1903-8.

5. Buechel RR, Husmann L, Pazhenkottil AP, Nkoulou R, Herzog BA, Burger IA, et al. Myocardial perfusion imaging with real-time respiratory triggering: Impact of inspiration breath-hold on left ventricular functional parameters. J Nucl Cardiol 2010;17:848-52.

6. Acampa W, Buechel RR, Gimelli A. Low dose in nuclear cardiology: State of the art in the era of new cadmium-zinctelluride cameras. Eur Heart J Cardiovasc Imaging 2016. doi: 10.1093/ehjci/jew036.

7. Friedman J, Van Train K, Maddahi J, Rozanski A, Prigent F, Bietendorf J, et al. "Upward creep", of the heart: A frequent source of false-positive reversible defects during thallium-201 stressredistribution SPECT. J Nucl Med 1989;30:1718-22.

8. Nakazato R, Berman DS, Hayes SW, Fish M, Padgett R, Xu Y, et al. Myocardial perfusion imaging with a solid-state camera: Simulation of a very low dose imaging protocol. J Nucl Med 2013;54:373-9. 